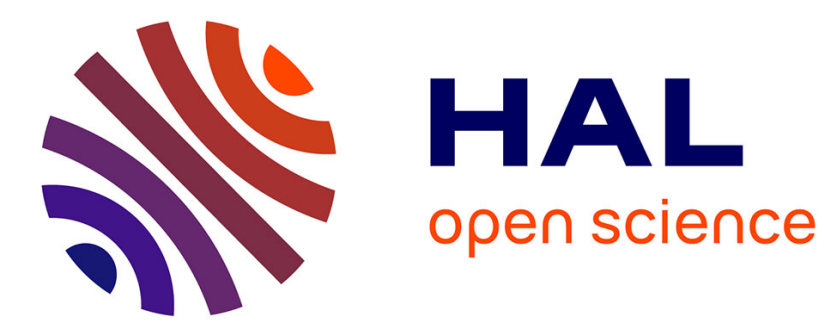

\title{
The Dynamics of Psychological Momentum: A Quantitative Study in Natural Sport Situations
}

Walid Briki, Ruud J.R. den Hartigh, Frank C Bakker, Christophe Gernigon

\section{To cite this version:}

Walid Briki, Ruud J.R. den Hartigh, Frank C Bakker, Christophe Gernigon. The Dynamics of Psychological Momentum: A Quantitative Study in Natural Sport Situations. International Journal of Performance Analysis in Sport, 2012, 12 (20), pp.573-592. 10.1080/24748668.2012.11868620 . hal02534557

\section{HAL Id: hal-02534557 \\ https://hal.science/hal-02534557}

Submitted on 7 Apr 2020

HAL is a multi-disciplinary open access archive for the deposit and dissemination of scientific research documents, whether they are published or not. The documents may come from teaching and research institutions in France or abroad, or from public or private research centers.
L'archive ouverte pluridisciplinaire HAL, est destinée au dépôt et à la diffusion de documents scientifiques de niveau recherche, publiés ou non, émanant des établissements d'enseignement et de recherche français ou étrangers, des laboratoires publics ou privés. 
See discussions, stats, and author profiles for this publication at: https://www.researchgate.net/publication/233728135

\section{The Dynamics of Psychological Momentum: A Quantitative Study in Natural Sport Situations}

Article in International Journal of Performance Analysis in Sport · December 2012 DOI: 10.1080/24748668.2012.11868620

\section{CITATIONS}

14

4 authors:

Walid Briki

Qatar University

51 PUBLICATIONS 422 CITATIONS

SEE PROFILE

Frank C. Bakker

Vrije Universiteit Amsterdam

63 PUBLICATIONS 2,758 CITATIONS

SEE PROFILE
Ruud J.R. Den Hartigh

University of Groningen

45 PUBLICATIONS 346 CITATIONS

SEE PROFILE

Christophe Gernigon

Université de Montpellier

70 PUBLICATIONS 915 CITATIONS

SEE PROFILE

Some of the authors of this publication are also working on these related projects:

Counterfactual Thinking and Imagination Page View project

Understanding Psychological Momentum View project 


\title{
The Dynamics of Psychological Momentum: A Quantitative Study in Natural Sport Situations
}

\author{
Walid Briki $^{1 *}$, Ruud J. R. Den Hartigh ${ }^{1,2^{*}}$, Frank C. Bakker ${ }^{3}$, Christophe Gernigon ${ }^{1}$ \\ ${ }^{1}$ Southern France Montpellier University, Department of Sport and Physical Education \\ Sciences, France \\ ${ }^{2}$ University of Groningen, Department of Psychology, The Netherlands \\ ${ }^{3}$ VU University Amsterdam, Faculty of Human Movement Sciences, Research Institute \\ Move, The Netherlands \\ * Both authors are considered as first authors.
}

\begin{abstract}
The present study examined the dynamics of competitive anxiety and selfconfidence, as well as the relationships between these variables, during athletes' experiences of psychological momentum (PM). Male national level table tennis players (Study 1) and swimmers (Study 2) watched one of their recent competitions, which included a PM experience. At the same time they indicated their moment-to-moment levels of competitive anxiety and selfconfidence by using a computer mouse. Curve estimation showed that competitive anxiety and self-confidence decreased and increased over time, respectively. Moreover, the dynamical patterns were more nonlinear for swimmers than for table tennis players, indicating that the PM experience is dependent on the sport context. Consistent with the opposite dynamics of competitive anxiety and self-confidence, correlation analyses revealed a strong negative relationship between these variables, suggesting that PM constraints the relationship between competitive anxiety and self-confidence. Findings of this study offer new insights into the dynamics of PM and its ecological embedment.
\end{abstract}

Key words: dynamical approach, affects, curve estimation, mouse paradigm, windowed correlation

\section{Introduction}

Psychological Momentum (PM) is a popular topic that has triggered the curiosity of psychology researchers for over three decades. Momentum is originally a Latin word, which literally means "movement" or "moving power". Related to this, early to most 
recent conceptualizations of PM assume it is a dynamical phenomenon. In 1981, Adler explained PM as "a state of dynamic intensity marked by an elevated or depressed rate in motion, grace, and success" (p. 29). Recently, Gernigon et al. (2010) defined PM as "a positive or negative dynamics of cognitive, affective, motivational, physiological, and behavioral responses (and their couplings) to the perception of movement toward or away from either an appetitive or aversive outcome" (p. 397).

Every conceptualization of PM stresses that it includes a number of psychological variables that change when individuals move toward, or away from, the goal to be reached (Adler, 1981; Gernigon et al., 2010; Markman and Guenther, 2007; Vallerand et al., 1988). Nevertheless, "traditional" research methods have not yet been able to provide insights into how PM continuously changes over time. Indeed, PM has mostly been studied at only three (e.g., Shaw et al., 1992; Stanimirovic and Hanrahan, 2004) or four (Perreault et al., 1998) measurement occasions, thereby providing little information about its dynamics. The intensity of PM has, however, been assumed to undergo changes (amplification or reduction) during a competition (Adler, 1981), an assumption that is consistent with earlier findings that changes in affect - a psychological content of a PM experience (e.g., Taylor and Demick, 1994) — are determined by perceived changes in the velocity of approaching a goal (Carver and Scheier, 1990; Hsee et al., 1994).

Recently, Gernigon and colleagues examined in more detail how various psychological variables change during momentum (Briki et al., in press; Gernigon et al., 2010). Gernigon et al. (2010, Experiment 1) studied the influence of a positive momentum scenario (i.e., winning ten points in a row) on the dynamics of competitive anxiety and self-confidence when participants empathized with a table tennis player on a screen. Cognitive anxiety-negative concerns about performance-and somatic anxiety-perceptions of bodily symptoms of competitive anxiety-were found to decrease linearly, while selfconfidence was found to increase linearly. Based on these results, Gernigon et al. (2010) concluded that positive PM develops gradually. Examining PM qualitatively, Briki et al. (in press) interviewed table tennis players and swimmers individually about PM experiences during an important competition. Results showed that positive PM was related both to increases in positive affect (e.g., self-confidence) and to decreases in negative affect (e.g., anxiety). Furthermore, positive PM appeared to emerge in response to an unexpected positive event or situation, which was more favorable than the current situation. This finding suggests that positive PM can suddenly emerge, and hence increase abruptly.

The above mentioned recent studies refined the understanding of PM by focusing on its dynamics (Briki et al., in press; Gernigon et al., 2010). However, one point about PM dynamics still needs to be clarified: On the one hand, Gernigon et al.'s (2010) quantitative results, obtained through an experimental design in which participants had to empathize with a player, suggested that positive PM changes gradually. On the other hand, Briki et al.'s (in press) qualitative results, stemming from actual experiences of PM, suggested that PM may emerge abruptly. Whereas a qualitative method is a proper way to study phenomena within natural contexts, quantitative methods are better suited to assess changes in psychological variables. However, to date no research has addressed the dynamics of 
PM quantitatively in a natural context. Hence, the first purpose of the present research was to gain more insights into the dynamics of PM by quantitatively assessing continuous changes in athletes' competitive anxiety and self-confidence during a sport competition.

Although both competitive anxiety and self-confidence are considered as important performance-related variables involved in PM (Adler, 1981; Cornelius et al., 1997; Gernigon et al., 2010; Miller and Weinberg, 1991), there is little consistent evidence on the relationship between these variables in sport-performance contexts. According to Martens et al. (1990), cognitive anxiety and self-confidence are bipolar constructs. In other words, negative concerns about performance would change in the opposite direction of feelings of self-confidence. In contrast, other authors considered these two variables to be distinct and orthogonal constructs (e.g., Woodman and Hardy, 2003). Examining this question, Gernigon et al. (2002) used a procedure adapted from Vallacher and colleagues' mouse paradigm (e.g., Vallacher et al., 2002). To be more specific, Gernigon et al. (2002) asked a volleyball player to continuously indicate her feelings of anxiety and self-confidence by moving a cursor on a computer screen. The researchers found that these variables could successively display a variety of patterns of relationships (i.e., positive, negative, and neutral) during very short periods in time. However, because earlier research revealed that PM is characterized by opposite fluctuations of competitive anxiety and self confidence (Gernigon et al., 2010), it is interesting to examine whether the relationships between these variables remain stable during PM. Therefore, the second purpose of the present research was to test the relationships between competitive anxiety and self-confidence during the experience of PM.

Given that the nature of a PM experience may depend on the sports context (Briki et al., in press), we studied the dynamics of, and relationships between, competitive anxiety and self-confidence during PM in both table tennis (Study 1) and swimming (Study 2). These two sports were chosen, because they have different characteristics: Table tennis involves competitions against one opponent, variation in players' actions, discrete feedback of performance, and requires attention, decision making, fine motor control, and discontinuous efforts because of the pauses between exchanges. Swimming, on the other hand, involves competitions against several opponents, repetitive movements, and necessitates intensive and continuous physiological energy expenditure. Moreover, table tennis and swimming lend themselves well for research applying a timely mouse paradigm procedure, because table tennis games and swimming races are often completed within a short time frame.

\section{Study 1: The dynamics of psychological momentum in table tennis}

Although PM can evolve either gradually or abruptly (Adler, 1981), Gernigon et al.'s (2010) findings revealed a gradual change in anxiety and self-confidence during positive PM. However, Gernigon et al.'s (2010) study was based on virtual situations rather than natural sport situations, which may have lessened the psychological impact of positive momentum. Consequently, while cognitive anxiety and somatic anxiety were expected to decrease during PM, and self-confidence was expected to increase, no hypotheses were 
stated about the rate of the changes. Finally, because of their hypothesized simultaneous decrease, cognitive and somatic anxiety should be positively related during positive PM. Furthermore, because self-confidence was expected to increase, this variable should be negatively correlated with both cognitive and somatic anxiety.

\subsection{Method}

\subsubsection{Participants}

Five French table tennis players, competing at national level, volunteered to participate in this study. They were recruited from several clubs in the south of France with the consent of their presidents and coaches. The average age of the participants was 22.4 years $(S D=$ $3.36)$, and on average they participated 5.2 years $(S D=3.27)$ in national level competitions. The participants were assured that their data would be treated confidentially.

\subsubsection{Procedure}

The protocol of the study was approved by the ethical committee, and consisted of the following three phases: Video recordings of the matches, identification of PM phases, and dynamical measurements of the variables under study.

The competitions were recorded with a digital camera placed on a tripod at a three-quarter angle behind the participant, thereby providing an optimal view of the match. From one to five days after the recordings, the study was carried out with each participant individually either in a room of the club or University. In both cases, a research device including two laptops was placed on a table. The first laptop was used for the measurements, while the second laptop was placed behind and above the first laptop, and showed the video of the competition.

Before starting the measurements, the researcher informed the participant about his interest in PM. Because the word momentum does not exist in French, the concept was explained by idiomatic expressions as "having the wind in one's sails" and "spiraling up". We chose such expressions to avoid a PM description including the psychological variables of interest, which could have influenced the participants' responses during the study. Then, each participant was asked to recall whether he had a PM experience in one of the recorded games. To facilitate his recall, the researcher presented the participant a sheet on which the scoring scenario for each match was shown. If the participant selected a specific game, he watched this game on the upper screen while listening to the surrounding sounds of the match with a headphone for a vivid re-experience. Next, the researcher asked whether or not the game really contained a PM experience. When the participant confirmed this question, he was asked to watch the whole game again, and to precisely identify when the PM experience started and ended.

Subsequently, the psychological variables under study were measured by using the mouse paradigm procedure (e.g., Vallacher et al., 2002), which was installed on the first laptop. With this procedure, the participant could indicate his moment-to-moment levels of the 
psychological state of interest, while watching the game on the screen of the second computer. The participant sat in front of both laptops with a computer mouse at the side of his dominant hand. First, he read the instructions on the first laptop, explaining how to use the computer mouse to indicate his moment-to-moment levels of a certain psychological state. Then, on a second screen the participant could practice using the mouse paradigm for about $30 \mathrm{~s}$. Moving the mouse entailed movements of a cursor along a 640-pixel horizontal axis at the bottom of the screen.

The exercise was followed by the actual measurements of the variables. Before each measurement, the participant read a short instruction on the first laptop, and had the opportunity to ask for clarification. He was then asked to place the cursor at the starting position, which was a thick mark at the middle of the axis. Subsequently, the participant executed the mouse procedure while watching a video, beginning $15 \mathrm{~s}$ before the start of his indicated PM phase, and ending $15 \mathrm{~s}$ after his indicated PM phase (unless the phase of PM ended within $15 \mathrm{~s}$ before the end of the game). The first $15 \mathrm{~s}$ aimed to give the participant the opportunity to move the cursor from its imposed initial position at the middle of the axis to the position corresponding to his actual state. The last $15 \mathrm{~s}$ aimed to prevent the PM experience from influencing the participant's response to the next item.

\subsubsection{Measures}

The moment-to-moment levels of the psychological variable under study were reflected by the continuously varying positions of the cursor on the horizontal axis between "not at all" at the left side ( 0 pixels) and "very much so" at the right side (640 pixels). The item of the variable was placed in the middle of the screen, above the horizontal axis. While the participant moved the cursor with the computer mouse to indicate his levels of the psychological variable in question, a program recorded the distance (in pixels) between the position of the cursor and the left end of the axis five times per second (i.e., $5 \mathrm{~Hz}$ ). In this way, a time series of distances was generated for the duration of the video. This procedure was repeated after the measurement of each variable, until all three variables-cognitive anxiety, somatic anxiety, and self-confidence-were assessed. The variables were successively presented in a random order for each participant.

To check the accuracy of the indicated PM experience, the actual measurement started with the presentation of a single item reflecting PM, which was "I am in a positive spiral". This item was constructed on the basis of a prevalent French expression highly related to the phenomenon of PM in both sport and everyday life. For all participants, the start of the PM experience corresponded exactly to an increase in the measured level of PM. The items of cognitive anxiety, somatic anxiety, and self-confidence were selected from the Competitive State Anxiety Inventory 2 Revised (CSAI-2R; Martinent et al., 2010), which is a French adaptation of the Cox et al.'s (2003) CSAI-2R. The cognitive anxiety item was "I am concerned about losing"; the somatic anxiety item was "My body feels tight"; and the selfconfidence item was "I feel self-confident". In Martinent et al.'s (2010) psychometric validation of the complete questionnaire, these items displayed satisfactory loadings on their respective factors (cognitive anxiety: .76; somatic anxiety: .64; self-confidence: .75). 


\subsubsection{Analyses}

For all participants, both the first and last 15 s (i.e., 75 data points each) were deleted, which means that the analyses were carried out on the data corresponding to the length of the PM experience. Each experience of PM had a different length: 32 s (i.e., 160 data points) for player A; $88 \mathrm{~s}$ (i.e., 440 data points) for player B; $59.2 \mathrm{~s}$ (i.e., 296 data points) for player C; $39.2 \mathrm{~s}$ (i.e., 196 data points) for player D; and $43.2 \mathrm{~s}$ (i.e., 216 data points) for player E. Before analyzing the data, time series were reduced to a common amount of 160 data points, thus matching the shortest series. These reductions were carried out proportionally over the total length of the time series to preserve the fluctuations of the variables over the entire PM phase. For instance, player B's time series of 440 data points, which was 2.75 (i.e., 11/4) times longer than player A's time series, was reduced by deleting seven out of every 11 data points over the entire time series.

Two kinds of analyses were performed in this study: Curve estimation and correlation analyses. First, we regressed the variables on time with a curve estimation procedure to fit the data points of each variable. Then, we analyzed the first-, second-, and third order trends of the regression function. The first-order trend accounts for the linear term (fixed ratio), whereas second-order (quadratic) and third-order (cubic) trends account for the nonlinear terms (one or two changes in the ratio, respectively). Equation 1 presents the form of the regression function:

$F(X)=B_{0}+B_{1} X+B_{2} X^{2}+B_{3} X^{3}$,

where $F(X)$ is the estimate of the value of the variable; $X$ is time; $B_{0}$ is the intercept; and $B_{1}$, $B_{2}$, and $B_{3}$ are the unstandardized regression coefficients for the linear-, quadratic-, and cubic terms, respectively.

Regularities and changes in the slope of the regression function of the variable in question were obtained by the derivative function of $F(X)$, reflected by equation 2 :

$F^{\prime}(X)=B_{1}+2 B_{2} X+3 B_{3} X^{2}$.

Positive, negative, and null derivatives indicate increasing, decreasing, and constant slopes of the regression function, respectively. That is, a positive and positively sloped derivative curve indicates that the slope of the regression function increases more and more. A positive and negatively sloped derivative curve indicates that the slope of the regression function increases less and less. A negative and positively sloped derivative curve indicates that the slope of the regression function decreases less and less. A negative and negatively sloped derivative curve indicates that the slope of the regression function decreases more and more.

Second, correlation analyses were run between the time series of cognitive anxiety, somatic anxiety, and self-confidence, both for the entire time series and within a moving window of data. This windowed correlation analysis (e.g., Boker et al., 2002) aimed to examine changes over time in the relationship between two variables. For the present use of this 
analysis, Pearson's coefficient was calculated within a window including a fixed number of data points of two time series. After the first correlation was computed, the window moved forward one data point and computed a second correlation, and so forth until the window had covered the whole series. The correlation coefficients thus obtained were plotted to reveal how the degree of correlation between the variables evolved over time. To ensure a sufficient number of observations for statistically powerful correlation analyses, and to permit the window to move forward a high number of steps, the width of the window was set at 41 out of the 160 data points.

\subsection{Results}

\subsubsection{Curve estimation}

The regression results deriving from the curve estimation procedure are displayed in Table 1, and the graphs of the regression and derivative functions are shown in Figure 1.

Regarding cognitive anxiety, negative linear and quadratic trends, as well as a positive cubic trend were significant $(p<.001)$. The linear trend accounted for $94.3 \%$ of the variance, to which the quadratic trend added $3.3 \%$, and the cubic trend added a negligible part. The graph shows that cognitive anxiety starts with a slow decrease, and then decreases more and more almost until the end of the PM phase. Finally, this decrease becomes nearly constant.

The dynamics of somatic anxiety included significant negative linear and quadratic trends, as well as a positive cubic trend $(p<.001)$. The linear, quadratic, and cubic trends accounted for $98 \%, 0.5 \%$, and $0.1 \%$ of the total variance, respectively. The graph shows that somatic anxiety decreases slowly at the start. Then, it decreases more and more almost until the end of the PM phase. Finally, this decrease becomes constant.

Concerning self-confidence, positive linear and cubic trends, as well as a negative quadratic trend were significant $(p<.001)$. The linear, quadratic, and cubic trends accounted for $87.5 \%, 4.9 \%$, and $2.3 \%$ of the total variance, respectively. The graph shows that, after starting with an abrupt increase, self-confidence increases less and less until two-third of the PM phase. Then, the rate of this increase stabilizes, after which it increases again until the end of the PM phase. 
Table 1. Summary of Curve Estimation Results for Cognitive Anxiety, Somatic Anxiety, and Self-Confidence in Table tennis.

\begin{tabular}{|c|c|c|c|c|c|c|c|c|}
\hline Variables & Dimensions & $R^{2}$ (cum.) & $R^{2}$ change & $F$ & $B_{0}$ & $B_{1}$ & $B_{2}$ & $B_{3}$ \\
\hline \multirow{3}{*}{$\begin{array}{l}\text { Cognitive } \\
\text { Anxiety }\end{array}$} & Linear & .943 & & $2596.98 * * *$ & 439.30 & -1.53 & & \\
\hline & Quadratic & .976 & .033 & $3149.84 * * *$ & 409.16 & -.41 & -.01 & \\
\hline & Cubic & .976 & .000 & $2124.26^{* * * *}$ & 405.05 & -.11 & -.01 & $1.9 \times 10^{-5}$ \\
\hline \multirow[t]{3}{*}{ Somatic Anxiety } & Linear & .980 & & $7595.81 * * *$ & 422.94 & -1.77 & & \\
\hline & Quadratic & .985 & .005 & $5301.98 * * *$ & 408.63 & -1.24 & -.00 & \\
\hline & Cubic & .986 & .001 & $3566.25 * * *$ & 405.28 & -.99 & -.01 & $1.6 \times 10^{-5}$ \\
\hline \multirow[t]{3}{*}{ Self-confidence } & Linear & .875 & & $1108.47 * * *$ & 247.20 & 1.91 & & \\
\hline & Quadratic & .924 & .049 & $960.98 * * *$ & 199.43 & 3.68 & -.01 & \\
\hline & Cubic & .947 & .023 & $926.18 * * *$ & 160.62 & 6.53 & -.06 & $1.8 \times 10^{-4}$ \\
\hline
\end{tabular}

Note. $B_{0}=$ intercept of the regression function; $B_{1}=$ unstandardized regression coefficient of the first order trend; $B_{2}=$ unstandardized regression coefficient of the second order trend; $B_{3}=$ unstandardized regression coefficient of the third order trend.

$* * * p<.001$. 


\section{— Raw Data - - - Regression Function …..... Derivative Function}

A

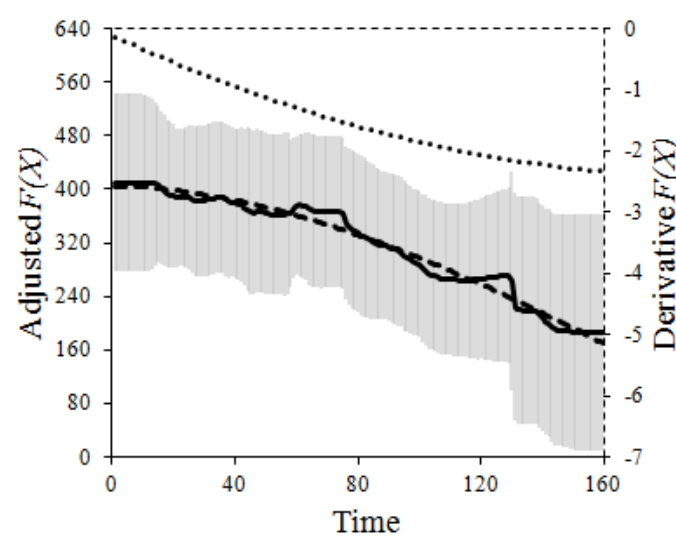

B

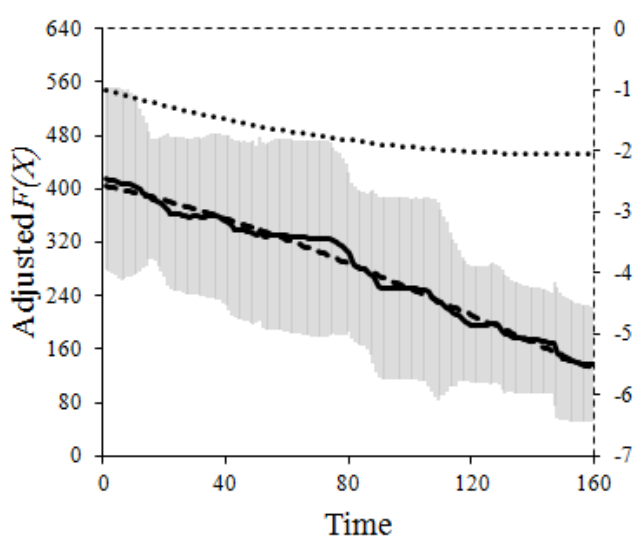

C

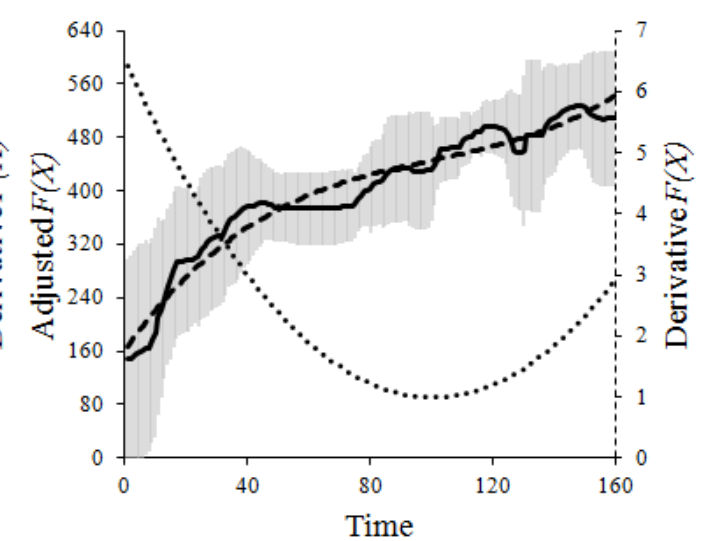

Figure 1. Trajectories of raw data (in pixels) according to time, regression function, and derivative function for cognitive anxiety (A), somatic anxiety (B), and self-confidence (C) in table tennis.

Note. The zero derivative value is marked with the horizontal dotted line. The grey area above and below the uninterrupted line represent the standard deviations of the raw data. 


\subsubsection{Interrelationships between variables}

Pearson correlation analyses revealed a high positive relationship between cognitive anxiety and somatic anxiety $\left(r_{(159)}=.98, p<.001\right)$. A high negative correlation was found between cognitive anxiety and self-confidence $\left(r_{(159)}=-.89, p<.001\right)$, as well as between somatic anxiety and self-confidence $\left(r_{(159)}=-.93, p<.001\right)$.

Concerning the relationships between the variables over time (see Figure 2), correlations between cognitive anxiety and somatic anxiety ranged from moderately positive $\left(r_{(40)}=.63\right.$, $p<.001)$ to highly positive $\left(r_{(40)}=.98, p<.001\right)$. The correlations between cognitive anxiety and self-confidence varied from highly negative $\left(r_{(40)}=-.98, p<.001\right)$ to moderately negative $\left(r_{(40)}=-.55, p<.001\right)$. The correlations between self-confidence and somatic anxiety ranged from highly negative $\left(r_{(40)}=-.98, p<.001\right)$ to moderately negative $\left(r_{(40)}=-.35, p<.001\right)$.

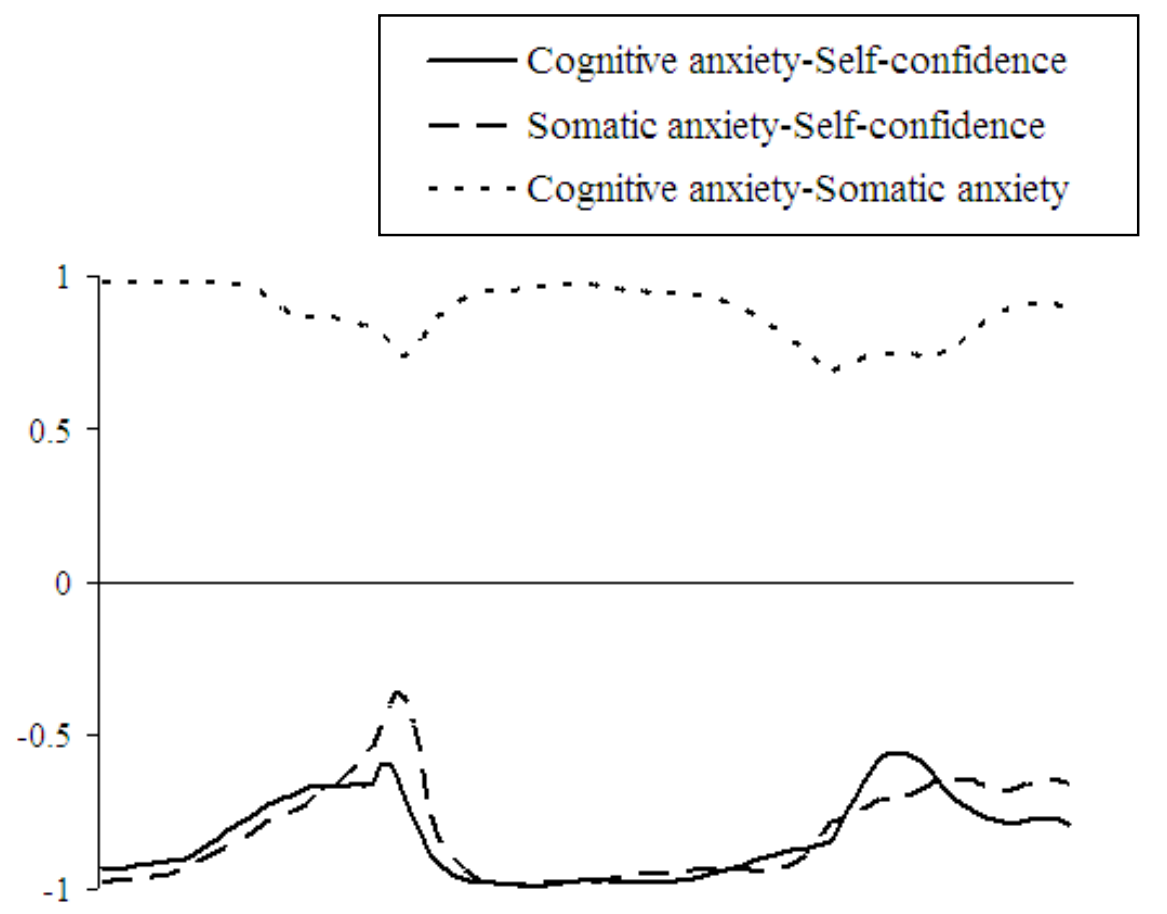

Figure 2. Relationship between cognitive anxiety and self-confidence, somatic anxiety and self-confidence, and cognitive anxiety and somatic anxiety over time in table tennis.

\subsection{Discussion}

One purpose of this study was to examine the dynamics of competitive anxiety and selfconfidence during PM experiences that were identified by table tennis players. Overall, 
cognitive and somatic anxiety decreased during PM, whereas self-confidence increased. These results support previous research findings that progressing toward a desired goal reduces competitive anxiety (Gernigon et al., 2010) and increases self-confidence (Gernigon et al., 2010; Shaw et al., 1992; Stanimirovic and Hanrahan, 2004).

Competitive anxiety and self-confidence showed mainly a linear dynamics, supporting previous research that found these variables to gradually change during PM (Gernigon et al., 2010). However, the dynamics of self-confidence also included slight quadratic and cubic trends, which can be explained by the abrupt increase at the start of the PM phase. Unlike competitive anxiety, self-confidence seems to react more strongly when gaining PM in an actual competition, compared to a virtual competition as in Gernigon et al.'s (2010) study. Nevertheless, regardless of the nature of the competition, competitive anxiety and self-confidence have been consistently found to change in specific directions, which supports the notion that competitive anxiety (Briki et al., in press; Gernigon et al., 2010) and self-confidence (e.g., Briki et al., in press; Gernigon et al., 2010; Shaw et al., 1992; Stanimirovic and Hanrahan, 2004) are variables characterizing PM.

The present study also aimed to examine how cognitive anxiety, somatic anxiety, and selfconfidence are interrelated during PM. Correlation analyses revealed strong positive relationships between cognitive anxiety and somatic anxiety, while their relationship over time ranged from moderately positive to strongly positive. Moreover, we found a strong negative relationship between self-confidence and the two forms of anxiety, and their relationship over time ranged from moderately negative to strongly negative. These findings shed a new light on the nature of the relationship between competitive anxiety and self-confidence (Martens et al., 1990; Gernigon et al., 2002). By consistently driving the levels of competitive anxiety and self-confidence downward and upward, respectively, the experience of PM maintains a negative relationship between these variables.

\section{Study 2: The dynamics of psychological momentum in swimming}

The previous study addressed PM in table tennis, a sport having its own unique characteristics. However, our ecological focus also requires an exploration of the dynamics of PM in a sport with different characteristics. Hence, this second study aimed to replicate Study 1 in swimming. Hypotheses were based on the findings of Study 1. First, cognitive anxiety and somatic anxiety were expected to decrease, whereas self-confidence was expected to increase. In addition, cognitive anxiety and somatic anxiety as well as selfconfidence were expected to be mainly characterized by linear dynamics. Moreover, both forms of anxiety were expected to gradually decrease during the entire PM experience, while self-confidence was expected to increase abruptly at the start of PM. Regarding the interrelationships among these variables, the two forms of anxiety were expected be positively correlated during PM, while they were expected to be negatively related to selfconfidence. 


\subsection{Method}

\subsubsection{Participants}

Four male swimmers, competing at national and international level, gave their consent to participate in this second study. The swimmers were specialists at the $50 \mathrm{~m}, 100 \mathrm{~m}$, and/or $200 \mathrm{~m}$. Their average age was 24.3 years $(S D=2.4)$, and on average they participated 4.75 years $(S D=1.26)$ at their current level. The participants were assured that their data would be treated confidentially.

\subsubsection{Procedure and measures}

Swimmers were asked whether they had a recent video of a race in which they had a PM experience. If we received a confirming response, an appointment was made to carry out the study in a room at the university. The procedure of the study was the same as in Study 1.

\subsubsection{Analyses}

The experiences of PM corresponded to $32 \mathrm{~s}$ (i.e., 160 data points) for swimmer A; $34 \mathrm{~s}$ (i.e., 170 data points) for swimmer B; $40 \mathrm{~s}$ (i.e., 200 data points) for swimmer C; and $58 \mathrm{~s}$ (i.e., 290 data points) for swimmer D. All time series were reduced to the length of the shortest time series, corresponding to 160 data points, following the same method as in the previous study. Subsequent analyses were identical to Study 1.

\subsection{Results}

\subsubsection{Curve estimation}

The regression results of the curve estimation procedure are summarized in Table 2, and the graphs of the regression and derivative functions are shown in Figure 3.

Cognitive anxiety displayed significant negative linear and cubic trends, as well as a positive quadratic trend $(p<.001)$. The linear, quadratic, and cubic trends accounted for $66.6 \%, 15.7 \%$, and $16.5 \%$ of the total variance, respectively. The graph shows that, after starting with an abrupt decrease, cognitive anxiety decreases less and less almost until the middle of the PM phase. Then, the level of this variable remains stable for a while before decreasing abruptly again at the end of the PM phase.

Regarding somatic anxiety, positive linear and quadratic trends, as well as a negative cubic trend were significant $(p<.001)$. The linear, quadratic, and cubic trends accounted for $28.6 \%, 46.5 \%$, and $8.9 \%$ of the total variance, respectively. The graph shows that somatic anxiety decreases less and less until the end of the first quarter of the PM phase. Next, it increases more and more almost until the end of the third quarter, to then finish quite stable. 
Table 2. Summary of Curve Estimation Results for Cognitive Anxiety, Somatic Anxiety, and Self-Confidence in Swimming.

\begin{tabular}{|c|c|c|c|c|c|c|c|c|}
\hline Variables & Dimensions & $R^{2}$ (cum.) & $R^{2}$ change & $F$ & $B_{0}$ & $B_{1}$ & $B_{2}$ & $B_{3}$ \\
\hline \multirow[t]{3}{*}{$\begin{array}{l}\text { Cognitive } \\
\text { Anxiety }\end{array}$} & Linear & .666 & & $315.66 * * *$ & 243.20 & -1.11 & & \\
\hline & Quadratic & .823 & .157 & $364.01 * * *$ & 299.82 & -3.21 & .01 & \\
\hline & Cubic & .988 & .165 & $4161.82 * * *$ & 370.00 & -8.36 & .09 & $-3.3 \times 10^{-4}$ \\
\hline \multirow[t]{3}{*}{ Somatic Anxiety } & Linear & .286 & & $63.42 * * *$ & 195.43 & .20 & & \\
\hline & Quadratic & .751 & .465 & $236.88 * * *$ & 222.27 & -.79 & .01 & \\
\hline & Cubic & .840 & .089 & $272.05 * * *$ & 236.38 & -1.83 & .02 & $-6.6 \times 10^{-5}$ \\
\hline \multirow[t]{3}{*}{ Self-confidence } & Linear & .240 & & $49.76 * * *$ & 468.97 & .47 & & \\
\hline & Quadratic & .450 & .210 & $64.20 * * *$ & 424.07 & 2.17 & -.01 & \\
\hline & Cubic & .972 & .522 & $1838.70^{* * * *}$ & 336.85 & 8.57 & -.11 & $4.1 \times 10^{-4}$ \\
\hline
\end{tabular}

Note. $B_{0}=$ intercept of the regression function; $B_{1}=$ unstandardized regression coefficient of the first order trend; $B_{2}=$ unstandardized regression coefficient of the second order trend; $B_{3}=$ unstandardized regression coefficient of the third order trend.

$* * * p<.001$. 
A

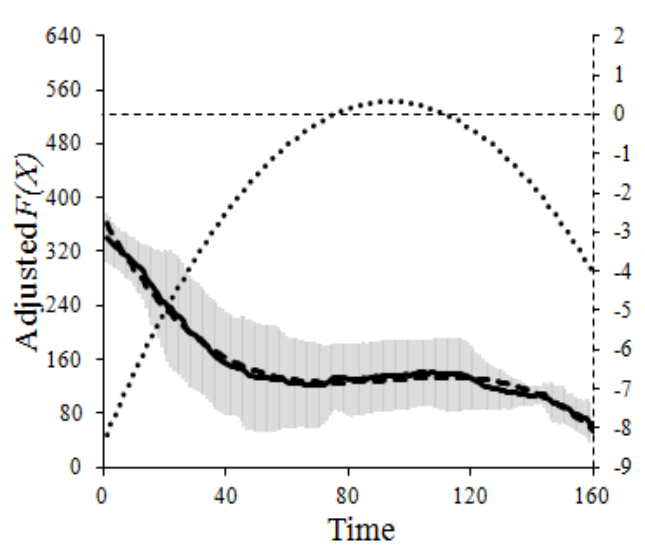

B

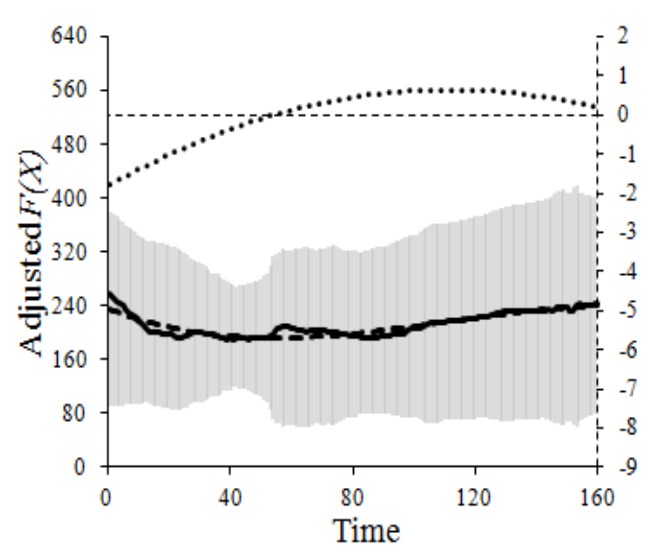

C

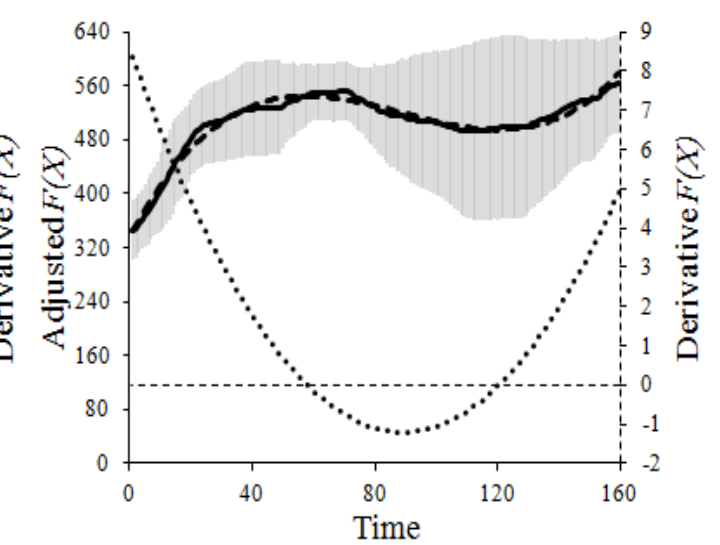

Figure 3. Trajectories of raw data (in pixels) according to time, regression function, and derivative function for cognitive anxiety (A), somatic anxiety (B), and self-confidence (C) in swimming.

Note. The zero derivative value is marked with the horizontal dotted line. The grey area above and below the uninterrupted line represent the standard deviations of the raw data. 
Self-confidence displayed significant positive linear and cubic trends, as well as a negative quadratic trend $(p<.001)$. The linear, quadratic, and cubic trends accounted for $24 \%, 21 \%$, and $52.2 \%$ of the total variance, respectively. The graph reveals that the increase of selfconfidence is abrupt at the start of the PM phase. Self-confidence then increases less and less until the end of the first third of the PM phase, after which a slight decrease sets in until the end of the third quarter. Then, self-confidence increases more and more until the end of the PM phase.

\subsubsection{Interrelationships between variables}

Pearson correlation analyses did not reveal a significant relationship between cognitive and somatic anxieties $(p>.05)$. A high negative correlation was found between cognitive anxiety and self-confidence $\left(r_{(159)}=-.88, p<.001\right)$, whereas a low negative correlation was found between somatic anxiety and self-confidence $\left(r_{(159)}=-.26, p<.001\right)$.

With regard to the relationship between the variables over time (see Figure 4), correlations between cognitive anxiety and somatic anxiety ranged from highly negative $\left(r_{(40)}=-.98, p\right.$ $<.001)$ to highly positive $\left(r_{(40)}=.83, p<.001\right)$. Correlations between somatic anxiety and self-confidence also ranged from highly negative $\left(r_{(40)}=-.94, p<.001\right)$ to highly positive $\left(r_{(40)}=.89, p<.001\right)$. The correlations between cognitive anxiety and self-confidence varied from highly negative $\left(r_{(40)}=-.97, p<.001\right)$ to non significant $(p>.05)$.

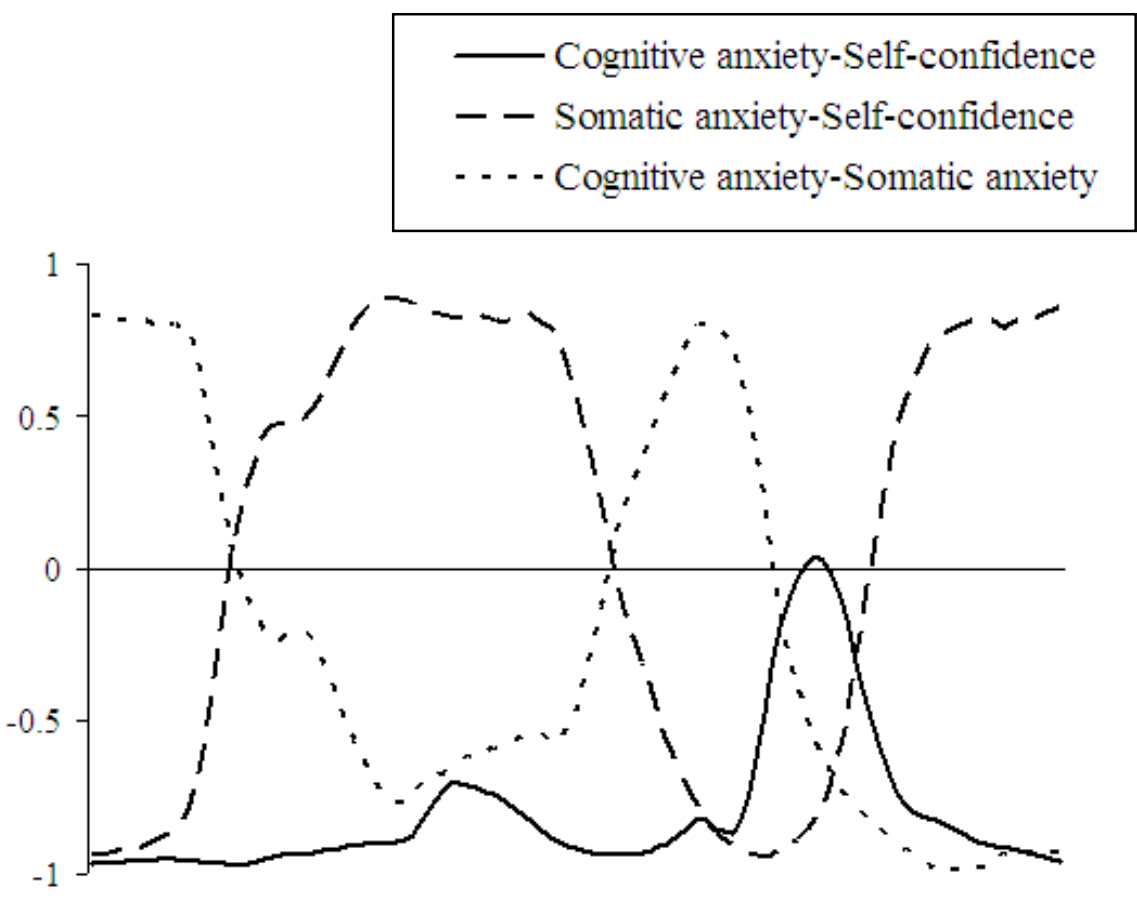

Figure 4. Relationship between cognitive anxiety and self-confidence, somatic anxiety and self-confidence, and cognitive anxiety and somatic anxiety over time in swimming. 


\subsection{Discussion}

This second study aimed to examine the dynamics of cognitive anxiety, somatic anxiety, and self-confidence during PM experiences of swimmers. Although the dynamics of cognitive anxiety was largely linear, quadratic and cubic trends also explained a considerable part of the variance. Cognitive anxiety displayed an abrupt decrease, which was followed by a phase of stability, and ended with another abrupt decrease. This pattern could be interpreted in light of the findings of Briki et al.'s (in press) qualitative study on PM. The first abrupt decrease might stem from a dissonance mechanism, causing a sudden emergence of PM. According to Briki et al. (in press), this mechanism corresponds to the perception of a discrepancy between an established situation or feeling (e.g., not feeling very strong) and an unexpectedly occurring event that is contradictory to that situation or feeling (e.g., realizing that one is ahead). Indeed, according to Carver and Scheier (1990) and Hsee et al. (1994), the more abrupt the change in the perceived rate of moving toward a goal, the greater and more sudden the change in affect. The stability phase may correspond to a period that Briki et al. (in press) called checking positive momentum, during which athletes assess whether their momentum is well established after its emergence. The swimmers in our study may have had some worries as long as they were not sure of their momentum. Then, once they noticed they had a comfortable lead, their worries could drop, which would explain the final abrupt decrease in cognitive anxiety.

The dynamics of somatic anxiety was mainly quadratic, but the linear and cubic trends also accounted for a fairly large and moderate part of the variance, respectively. Somatic anxiety started with a slight decrease until the end of the first quarter of the PM phase, followed by a slight increase till the end of the PM phase. This finding is not consistent with the hypothesized linear dynamics, nor the continuous decrease of somatic anxiety as found by Gernigon et al. (2010). The inconsistency might be due to the fact that Gernigon et al.'s (2010) experiment was based on virtual experiences of PM, whereas the present study was based on actual PM experiences. Yet another plausible reason is the ambiguity of the somatic anxiety item, which referred to body tightness. In swimming, body tightness may be caused by both physiological fatigue and anxiety; thus the moment-to-moment levels of the swimmers' indicated somatic anxiety might have reflected either their fatigue or their somatic anxiety. Hence, to capture somatic anxiety in swimming, a focus on other physiological manifestations of this variable, such as feeling that "one's stomach is sinking" (Cox et al., 2003), may be better suited.

The self-confidence dynamics was mostly cubic, and to a lesser extent explained by linear and quadratic trends. Three phases could be distinguished: An abrupt increase, a phase of stability, and another abrupt increase. It is remarkable that the graphs of cognitive anxiety and self-confidence are nearly symmetrical. Therefore, the same explanations that were provided for the dynamics of cognitive anxiety could also apply to the dynamics of selfconfidence.

Another purpose of the present study was to examine the interrelationships between the variables under study. Consistent with the symmetry between the graphs of cognitive 
anxiety and of self-confidence, the general relationship between these variables was strongly negative. Over time, the relationship between cognitive anxiety and selfconfidence ranged from strongly negative to neutral. It should, however, be noted that the neutral relationship was only shortly present around the end of the phase that both cognitive anxiety and self-confidence remained nearly stable. This may not come as a surprise, because the variables did not show a clear increase or decrease at that time. Nevertheless, a strong negative relationship was found between cognitive anxiety and self-confidence over almost the entire PM phase. This is in line with the results of Study 1, and supports the assumption that the experience of PM maintains negative relationships between anxiety and self-confidence by driving their dynamics downward and upward, respectively.

Contrary to our expectations, cognitive anxiety and somatic anxiety were not significantly correlated, and their relationship over time ranged from highly negative to highly positive. Moreover, the general relationship between somatic anxiety and self-confidence was moderately negative, while their relationship over time also ranged from highly negative to highly positive. These findings are not consistent with those of Study 1 nor with Gernigon et al. (2010), who showed similar changes in cognitive and somatic anxiety during PM, which were in the opposite direction of the changes in self-confidence. Again, these results remain difficult to interpret due to the aforementioned ambiguity of the somatic anxiety item we used.

\section{General discussion}

The purposes of the present research were to examine both the dynamics of, and the relationships between, competitive anxiety and self-confidence during PM experiences in natural sport settings. We found evidence that positive PM is a dynamical phenomenon. In general, competitive anxiety decreased and self-confidence increased, which supports previous research showing similar changes in competitive anxiety (Gernigon et al., 2010) and self-confidence (Gernigon et al., 2010; Shaw et al., 1992) during PM.

Beyond the general trends, self-confidence was found to change abruptly for both table tennis players and swimmers, as soon as PM was triggered. The high sensitivity of selfconfidence to PM whatever the type of activity supports the view that self-confidence is one of the key variables of PM (Jones and Harwood, 2008; Taylor and Demick, 1994). Cognitive anxiety also changed abruptly at the start of PM, but only in swimming. The difference between table tennis players and swimmers with regard to the dynamics of anxiety indicates that the dynamics of PM may differ for different sports (Briki et al., in press). The more abrupt change in cognitive anxiety for swimmers at the start of PM might be due to the fact that gaining momentum generates stronger expectancies of future success in a swimming race. To be more specific, one cannot be stuck on the spot while the opponents advance in a swimming race. In table tennis, on the other hand, one can head towards the win, but then gain no more points while the opponent scores more and more points and catches up. 
More generally, for table tennis players the dynamics of the variables under study were mainly linear, whereas more nonlinear dynamics were observed for swimmers. The greater nonlinearity we found in the swimming study could be due to the lack of comparison feedback that swimmers receive during the race. Whereas table tennis players are informed of the scoring point after point, swimmers can only get a clear picture of their position in the race when turning, or when making an effort to look at the neighboring lanes. As a result, having gained momentum swimmers would experience a greater uncertainty about their position in relation to the opponents, compared to table tennis players. This prompts the swimmers to engage in the checking behaviors as found by Briki et al. (in press), and lead to a temporal cease in the (abrupt) decrease and increase in their worries and feelings of confidence, respectively. Overall, these findings evidence that the experience of PM is ecologically embedded (Briki et al., in press).

Considering the relationships between the variables under study, cognitive and somatic anxieties were strongly positively related in the first study, whereas these variables were found to relate strongly negatively to self-confidence. In the second study, cognitive anxiety and self-confidence showed a strong negative correlation as well. Besides, with the exception of a short period of a non significant relationship between cognitive anxiety and self-confidence in Study 2, both studies revealed the mentioned kinds of relationships from the start until the end of the PM phase. Therefore, compared to other sport situations in which the relationship between anxiety and self-confidence undergoes abrupt variations (Gernigon et al., 2002), the experience of PM seems to constraint the relationship between these variables.

This research presents the first attempt to quantitatively capture the dynamics of PM in actual sport competitions. However, two limitations should be addressed. First, because the mouse paradigm procedure is a retrospective method it carries the risk of recall bias. However, to minimize this bias, the measurements were carried out as soon as possible after the recorded competitions. Second, the somatic anxiety item we used may not have clearly distinguished body tightness due to somatic anxiety from body tightness due to exerted effort and fatigue.

To conclude, the present findings incite to pursue the examination of PM dynamics in actual sport performance settings. Further studies need to be carried out on other dimensions of PM, such as perceptions and cognitions, which are also involved in PM (Briki et al., in press). Finally, it would be fruitful to examine the dynamics of negative PM because recent research suggests that positive and negative PM are characterized by specific dynamics (Briki et al., in press; Gernigon et al., 2010). 


\section{References}

Adler, P. (1981), Momentum: A theory of social action. Beverly Hills, CA: Sage.

Boker, S. M., Xu, M., Rotondo, J. L. and King, K. (2002), Windowed cross-correlation and peak picking for the analysis of variability in the association between behavioral time series. Psychological Methods, 7, 338-355.

Briki, W., Den Hartigh, R. J. R., Hauw, D. and Gernigon, C. (in press), A qualitative exploration of the psychological contents and dynamics of momentum in sport. International Journal of Sport Psychology.

Carver, C. S. and Scheier, M. F. (1990), Origins and functions of positive and negative affect: A control-process view. Psychological Review, 97, 19-35.

Cornelius, A., Silva, J. M., Conroy, D. E. and Peterson, G. (1997), The projected performance model: Relating cognitive and performance antecedents of psychological momentum. Perceptual and Motor Skills, 84, 475-485.

Cox, R. H., Martens, M. P. and Russel, W. D. (2003), Measuring anxiety in athletics: The revised competitive state anxiety inventory-2. Journal of Sport \& Exercise Psychology, 25, 519-533.

Gernigon, C., Briki, W. and Eykens, K. (2010), The dynamics of psychological momentum in sport: The role of ongoing history of performance patterns. Journal of Sport \& Exercise Psychology, 32, 377-400.

Gernigon, C., Yvelin, N., Delignières, D., Ninot, G. and d'Arripe-Longueville, F. (2002), A dynamic approach to the variations in the perception of control, anxiety, and selfconfidence, and in their patterns of synchronization during time-out in sport. Journal of Sport \& Exercise Psychology, 24, S62.

Hsee, C. K., Salovey, P. and Abelson, R. P. (1994), The quasi-acceleration relation: Satisfaction as a function of the change in velocity of outcome over time. Journal of Experimental Social Psychology, 30, 96-111.

Jones, M. I. and Harwood, C. (2008), Psychological momentum within competitive soccer: Players' perspectives. Journal of Applied Sport Psychology, 20, 57-72.

Markman, K. D. and Guenther, C. L. (2007), Psychological momentum: Intuitive physics and naive beliefs. Personality and Social Psychology Bulletin, 33, 800-812.

Martens, R., Vealey, R. S. and Burton, D. (1990), Competitive anxiety in sport. Champaign, IL: Human Kinetics.

Martinent, G., Ferrand, C., Guillet, E. and Gautheur, S. (2010), Validation of the French version of the Competitive State Anxiety Inventory-2 Revised (CSAI-2R) including frequency and direction scales. Psychology of Sport and Exercise, 11, 51-57.

Miller, S. and Weinberg, R.S. (1991), Perceptions of psychological momentum and their relationship to performance. The Sport Psychologist, 5, 211-222.

Shaw, J. M., Dzewaltowski, D. A. and McElroy, M. (1992), Self-efficacy and causal attributions as mediators of psychological momentum. Journal of Sport \& Exercise Psychology, 14, 134-147.

Stanimirovic, R. and Hanrahan, S. J. (2004), Efficacy, affect and teams: Is momentum a misnomer? International Journal of Sport and Exercise Psychology, 2, 43-62.

Taylor, J. and Demick, A. (1994), A multidimensional model of momentum in sports. Journal of Applied Sport Psychology, 6, 51-70. 
Vallacher, R. R., Nowak, A., Froehlich, M. and Rockloff, M. (2002), The dynamics of selfevaluation. Personality and Social Psychology Review, 6, 370-379.

Vallerand, J. R., Colavecchio, P. G. and Pelletier, L. G. (1988), Psychological momentum and performance inferences: A preliminary test of the antecedents-consequences psychological momentum model. Journal of Sport \& Exercise Psychology, 10, 92-108.

Woodman, T. and Hardy, L. (2003), The relative impact of cognitive anxiety and selfconfidence upon sport performance: A meta-analysis. Journal of Sports Sciences, $21,443-457$. 